\title{
Understanding the Characteristics of the Scalp for Developing Scalp Care Products
}

\author{
Sehyun Kim, Seunghyun Shin, Su-Na Kim, Yongjoo $\mathrm{Na}^{*}$ \\ Amorepacific R\&D Center, Gyeonggi-do, Republic of Korea \\ Email: ^nay@amorepacific.com
}

How to cite this paper: Kim, S., Shin, S., Kim, S.-N. and Na, Y. (2021) Understanding the Characteristics of the Scalp for Developing Scalp Care Products. Journal of Cosmetics, Dermatological Sciences and Applications, 11, 204-216.

https://doi.org/10.4236/jcdsa.2021.113018

Received: June 28, 2021

Accepted: August 10, 2021

Published: August 13, 2021

Copyright (อ 2021 by author(s) and Scientific Research Publishing Inc. This work is licensed under the Creative Commons Attribution International License (CC BY 4.0).

http://creativecommons.org/licenses/by/4.0/ (c) (i) Open Access

\begin{abstract}
With the exception of conditions such as dandruff, interest in the scalp is relatively low. However, when we consider the scalp as part of the skin, interesting observations can be made. The average amount of sebum secretion in the scalp varies depending on sex. Several studies show differences in scalp characteristics among races, but in some cases, these are difficult to find. The scalp has a weaker barrier function than does facial skin. The reason for the weak barrier function is unclear, but it is necessary to consider the effect of dandruff on the scalp. Growth of dandruff-related bacteria weakens the barrier. However, strengthening the barrier suppresses dandruff. In other words, there is an interdependency between scalp bacteria and skin, which is important for the maintenance of scalp barrier homeostasis. Anxiety regarding a sensitive scalp is as high as that for sensitive skin. At least $40 \%-50 \%$ of individuals in modern society have complaints such as itching due to a sensitive scalp. The sensitivity of the scalp is similar between races. This is not a skin disease, but we have to think about how problems that customers face can be solved. Skin barrier and sensitive scalp are closely related. Collectively, strengthening or lowering the effect on the scalp barrier may result in a healthy scalp environment. As a result, it will alleviate people's complaints about the scalp.
\end{abstract}

\section{Keywords}

Scalp Care Products, Sensitivity, The Scalp Barrier

\section{Introduction}

The hair is an important attraction factor for both men and women [1] [2]. In order to maintain good quality of hair, the cosmetic industry has developed several hair cleansing and conditioning products. However, from the perspective of 
fundamentally managing the quality of hair, we need to pay attention to the scalp. The scalp condition affects the quality of hair [3]. It is true that "healthy hair is from healthy scalp".

Nevertheless, interest in the scalp is limited. The scalp has received much attention in terms of diseases such as dandruff and seborrheic dermatitis. The cause of the disease has been well studied [4]. In the case of dandruff, appropriate solutions such as zinc pyrithione (ZPT) [5] have also been developed and are widely applied in commercial shampoos. However, research on the scalp as skin, not a disease, is inadequate.

There is more reason to be interested in the scalp. The scalp has an environment where it is easy to get dirty and microorganisms grow well [6]. Although this may not be a serious disease, it is an important clue to solve the beauty grievances that people may experience. It is necessary to manage the scalp skin well as people manage facial skin. It is also worth considering from the perspective of quality of life. Recently, interest in sensitive skin is increasing due to environmental pollution and urbanized life. The scalp is no exception to this situation.

From this point of view, we would like to point out what needs research and development for effective scalp care. However, we will exclude ecosystems of scalp microorganisms because they have already been studied extensively. In this review, basic characteristics of the scalp, studies on barrier function, and sensitive scalp will be discussed. At the end of these discussions, we would like to suggest the direction of developing cosmetics for healthy scalp.

\section{Basic Features of Scalp: Sex and Races}

The skin structure of the scalp is similar to that of other body parts. The biggest feature that characterizes the scalp is the large hair follicle with terminal hair [7]. The scalp is also vascularized and has more sebaceous glands and sweat glands than other skin areas [8]. Many sebaceous glands provide a favorable environment for various types of bacteria [9] [10] and cause diseases that are specific to the scalp. From an aesthetic point of view, it could become dirtier than other skin areas. Nonetheless, normal skin is easy to recognize, making it easier to treat and care faster, while the scalp is difficult to recognize the problem on its own. Therefore, the scalp should be managed more carefully.

The degree of proliferation of normal epidermal cells tends to be inversely proportional to the size of corneocytes (dead epidermal cells). The smaller the keratinocyte size, the faster the keratinous turnover rate. For example, corneocyte size increases with age [11]. A study showed that the keratinocyte size of the scalp was significantly larger than that of the face and smaller than that of the fore arm. Its turnover rate is slower than that in the facial skin but faster than that in the arm [12].

According to a report released by Unilever in 2017 (not published, presented at 12th World Congress of Cosmetic Dermatology), there is a difference between 
men and women's scalp. Although it is an observation of the phenomenon, there were some interesting points in their internal studies. It is a study that collects and analyzes at least 1000 clinical data from major continents and countries (Brazil [1002], Turkey [989], Russia [1010], India [3208], China [2006], Indonesia [1224], and Japan [1127]). There is more protein loss in men's scalp. The average amount of sebum in the scalp of men is about 1.5 times higher than that of women. This sebum amount result is consistent with that from another study performed by L'Oreal [13]. There is less amount of ceramide present in the horny layer of men's scalp. Ceramide is a component of the stratum corneum and plays an important role in skin barrier function [14] [15]. That is, the skin barrier function of men's scalp is weaker than that of women. This phenomenon is linked to men being more susceptible to dandruff. According to this study, men are more likely to have dandruff and itchy and oily scalp than women. A meta-analysis of the Bangkok studies further showed that men are more responsive to anti-dandruff shampoos.

P\&G conducted a similar study. However, clinical data from 1114 subjects were evaluated, and there was no statistically significant difference in biomarkers between the sexes, including flaking of the scalp. There were no significant results in the Asian and White clinical subgroups in the analysis of race-dependent differences. As a result, the efficacy of ZPT shampoos is irrelevant to sex and ethnicity [16]. Thus, the efficacy of ZPT shampoo can be interpreted to be meaningful to both men and women. The conclusions drawn from L'Oreal's study on differences in sex-specific ZPT response differ from those obtained from Unilever's previous study. The evaluation indexes measured in each study are different. Total weighed head score reduction was measured in the Unilever study, and adherent scalp flake score grading was measured in the P\&G study. Therefore, this should be reflected in understanding the results of the male-female response to the anti-dandruff shampoo.

There have been some studies on the difference in skin characteristics between races [17]. Because of the nature of the scalp, mainly sebum-based racial comparison studies were performed. Human sebum is regulated by hormones, which increase in puberty. The secretion of sebum is maintained at $20-30$ years and decreases with age [18]. If so, will there be differences in race and ethnicity? A total of 41 British and 31 Thai individuals (each dry/wet season) were recruited in order to identify the differences in dandruff between races. In subjects with dandruff, the amount of fatty acids, cholesterol, and ceramides decreased, regardless of race. However, the overall level of scalp lipid was similar in English and Thai people [19]. Researchers did not find a large difference in sebum secretion between races in this study.

Let us look at the results of a recent study. It covers the wide range of racial differences across the globe from 2007 to 2011 (Pudong and Shanghai, China, October 2007; Clark, Chicago, USA, April 2009; Bangkok, Thailand, May 2009; Paris, France, October 2009; Kawasaki and Tokyo, Japan, December 2010; 
Mumbai, Chennai, Delhi, and Kolkata, India, 2011) [13]. There was a limitation with respect to the consistency of these experiments and the controls used because they were carried out separately, for long durations, and different regions were examined. However, from a statistical point of view, there is value as a research that can confirm the difference on scalp sebum between races shortly after shampooing and after a certain time. Sebum secretion curves showed a hyperbolic shape after the scalp was regreased (after sebum secretion). Shampoo habits in the subjects of this study were found to affect the basic amount of sebum (measured within $1 \mathrm{~h}$ after shampooing). The average number of shampoos per week is 1.9 in African American, 3 in Indian, 3.5 in Chinese, 3.8 in French, 4.2 in Thai, and 4.7 in Caucasian American people. African American individuals, who had the least frequent hair shampooing a week, had the highest amount of basic sebum. All other groups with an average of three or more hair shampooing per week had less than half of their basic lipid amount compared to African American people. This phenomenon also affects the level of self-perception of oily hair by the subject themselves. The interesting result is that there is a difference in the average amount of scalp sebum between races, and that overall amount of sebum is quite low in Indian people. Although there are some claims that shampooing often leads to increased sebum secretion [20] [21], at least in this study, it is suggested that frequent use of shampoo can control scalp sebum secretion.

\section{Scalp Barrier Function}

Generally, transepidermal water loss (TEWL) was measured as an index of skin barrier function [22] [23]. An increase in TEWL means that the skin barrier function is impaired [24]. TEWL may vary depending on the site or period [25]. We can use TEWL values to compare relative barrier functions of each skin area. The average TEWL of the scalp is higher than that of the arm or back [12] [26].

Interestingly, the scalp barrier function is also related to the hair loss phenomenon. Researchers also examined differences between hair loss and non-hair loss areas. In a study of patients with hair loss [12], the TEWL of the scalp was higher. A study in 1972 [27] analyzed the thicknesses between the general area of the scalp and the hair loss area. The thickness of each layer of the scalp gradually increases and decreases with age, depending on the region and sex. According to the degree of hair loss, the thickness of the epidermis, dermis, and subcutaneous fat of the scalp decreased as the amount of hair loss increased. These features are consistent with epidermal/dermal thickness in studies conducted on the Korean population (written in Korean) [28]. Histopathological findings from lesions of 20 patients with alopecia were compared, and it was found that the stratum corneum and epidermis were significantly thinner in those with alopecia.

Overall, the scalp has relatively less barrier function than other skin areas. Unfortunately, there is no clear explanation on why the scalp skin barrier is weak. In the meantime, we can conclude that the special conditions of the scalp 
are related to barrier function by summarizing the studies. It is highly related to the formation of influential microorganisms in the scalp, function of well-developed sebaceous glands, and composition of sebum. Several studies of scalp skin barriers provide hints on this issue.

A scalp with dandruff exhibits higher TEWL than a healthy scalp [29]. When the scalp skin barrier is weakened, a vicious cycle occurs in which vulnerability to dandruff increases [30]. The amount of natural moisturizing factor (NMF) was lower in the dandruff group than in the normal group [31]. Generally, it is expected that the scalp is moisturized by hair and scalp sebum. NMF is a collection of water-soluble, low-molecular components that effectively absorb moisture in the air to provide moisturization [32]. Low NMF levels are often observed in dry skin, atopic dermatitis, and psoriasis, which are typical skin diseases [33]. In other words, low NMF levels reflect poor skin health. When ZPT-containing antibiotics were used to remove dandruff, the scalp barrier functions including NMF were improved or normalized [31]. This fact suggests that dandruff may be a major contributor to scalp skin barrier function.

Interestingly, restoration of epidermal function improves dandruff and restores scalp homeostasis. In this study, the researchers used a plant-derived material and applied it to the scalp. The effect of improving the barrier function of the scalp and relieving inflammation has resulted in a significant improvement in clinical dandruff index [34]. As mentioned in the results of the study, it is not only the improvement of the skin barrier due to the treatment with the ingredient but also the ability to inhibit the fungi Malassezia to a certain extent. There is a limit to conclude that restoration of skin barrier function leads to dandruff improvement. However, it is clear that skin barrier function has a significant relationship with the development of and reduction in dandruff.

We can also find clues to associations of scalp barrier function with lipid characteristics found in scalp with dandruff. In the case of a scalp with dandruff, the required lipid content of the stratum corneum decreased, and the relative ratio also changed. Dry scalp symptoms are often observed in patients with atopic dermatitis, seborrheic dermatitis, or pityriasis capitis [35]. In these patients, scalp lesions show reduced ceramide levels with low barrier function. The following experiment was conducted on 30 Japanese women with diseases such as atopic dermatitis and seborrheic dermatitis. Lotions containing synthetic ceramide (cetyl-PG hydroxyethyl palmitamide) and eucalyptus extracts were applied daily to the scalp after shampooing. As a result, there was improvement in scalp condition 2 weeks after application. Especially, scaling, erythema, and itching were significantly improved. The skin barrier function of the scalp was greatly improved [36]. Although this study was performed on patients with certain skin disorders, its results are also of value in the general population with weak scalp skin barriers.

Barrier strengthening improves the scalp condition directly or indirectly and alleviates sensitivity to external stimuli. What target would be effective for enhancing or maintaining the homeostasis of the skin barrier? Skin barrier func- 
tion can be maintained well by preventing intrusion by foreign substances and maintenance of internal substances. Several factors responsible for skin barrier function are well known, but it is the tight junction (TJ) that plays a physically important role [37]. TJ is a junction between cells. Therefore, it functions to restrict transfer of various sized molecules between cells. Damage to the TJ increases the TEWL even though the stratum corneum is present. These results can be confirmed by experiments such as knockdown of proteins constituting the TJ [38] [39]. TJs are expressed in not only the epidermal granules but also follicular root (outer root sheath) [37]. There is a limitation to the experiment using porcine skin hair follicles rather than human hair follicles. However, we can understand the role of $\mathrm{TJ}$ as a barrier in the hair follicles. Compartmentalization of the follicles is needed in areas that reflect the accessibility of molecules entering hair follicles. Molecules that enter the hair follicle must pass through two barriers, the TJ and stratum corneum at the top, while the $\mathrm{TJ}$ is the only barrier at the bottom. However, the experiment using nanoparticles showed that most of the particles were located in the upper part of the hair follicle, in which the stratum corneum and TJ barrier coexist. TJ in the hair follicle also prevents the entry of foreign substances but also plays a role in preventing the loss of nutrients and hormones in the hair follicles [40]. Thus, we could suggest a TJ as a suitable target for maintaining and enhancing skin barrier function for the scalp. The role of the $\mathrm{T} J$ is very important from the viewpoint of the skin barrier of the scalp, and a TJ strengthening strategy is also needed to prevent external factors penetrating through hair follicles.

\section{Sensitive Scalp}

Sensitive skin is a term that is used in the cosmetic industry to refer to a case where skin problems are caused by use of cosmetics and the like [41]. It has long been a concern for consumers and the cosmetic industry. It has been linked to recent changes in the living environment, air pollution, changes in diet, and stress. It may be caused by various factors such as ultraviolet radiation, heat, cold, and wind, i.e., physical environment. It may also be caused by chemical (cosmetics, soap, water, and pollution), psychological (stress), or hormonal (menstrual cycle) factors [42].

Sensitive skin or scalp was the term used by laypersons, but academic studies using these terms were published in 2008. Pierre Fabre introduced a study of sensitive scalp with more than 1000 French men and women [43]. In this study, $47.4 \%$ of French women and $40.8 \%$ of French men revealed that they had sensitive scalp, and $44.2 \%$ of people had sensitive scalp. Of the respondents with a sensitive scalp, $11.5 \%$ actually had scalp disease (versus $1.1 \%$ of the non-sensitive group). When the scalp was classified as dry/normal/intelligent, the proportion was $24 \%, 58 \%$, and $16 \%$ of the subjects, respectively. About $66 \%$ of the normal scalp is not sensitive or slightly sensitive, while $60 \%$ of the dry scalp is sensitive or very sensitive. The percentage of oily scalp was similar. This means that the 
frequency of grievances actually felt by the customer differs depending on the condition of the scalp.

In 2015, a study of sensitive scalp skin in Chinese women was published [44]. The purpose of this study was to develop a questionnaire to more accurately determine the self-awareness of sensitive scalp. Although the response rate varies according to the self-questionnaire method for sensitive scalp, 36\% - 57\% of Chinese women said they have a sensitive scalp by themselves. As a result, dryness, exercise, humidity, and heat factors or conditions have been found to play a significant role in itchy sensitive scalp. Unfortunately, this study was limited to the winter season; thus, there are limits to the interpretation.

According to a previous study [43], a quarter of French people have very common scalp complaints of itchy scalp. Sensitive scalp is a symptom of noticeable irritation or discomfort to the scalp without immune response. The incidence of sensitive scalp varies in studies but is about $30 \%$ to $40 \%$. Of patients with sensitive scalp, $60 \%$ complain of itchiness [45], and scalp sensitivity is associated with hair loss [43]. Since itchy scalp is a big problem for customers, we need to figure out more about the reason for this phenomenon. Three aspects of itchy scalp can be explained: dermatological, pathophysiological, and scalp-specific factors [46].

Seborrheic dermatitis is the most common complication of scalp itching. Although depending on the studies [47] [48], at least $60 \%$ of patients with psoriasis also complain of scalp itching. There was a report that a positive correlation between severity of scalp itching and psoriasis [48]. Fungi Malassezia causes inflammation by the breakdown of sebum, and histamine secretion is two times higher in seborrheic dermatitis skin. Scalp has a complex nerve structure. Around the hair follicles, nerves, tactile sense, and pressure is present. Does the scalp feel itchier than the other skin areas? There are some interesting results. When histamine is injected, the entire arm of the skin becomes more itchy than the scalp, but when it is applied to the skin topically, the scalp becomes more itchy [49]. The scalp is less sensitive to heat stimuli than to other areas. In other words, the scalp contains several nerve fibers, but it is not particularly sensitive to other skin areas [50]. However, at least this is clear: antibacterial ZPT shampoo reduces histamine levels as well as dandruff, and patients' perception of scalp itching has also improved [51].

There is a high density of Malassezia on the scalp. IL-6, IL-8, and IL- $1 \alpha$ secretion was increased in skin cells when lipid components of Malassezia cell wall were extracted [52]. The lipase of Malassezia decomposes the triglyceride of scalp sebum to produce free fatty acids. It is known that unsaturated fatty acid consuming certain saturated fatty acid stimulates the skin [53]. The role of sebum in the skin has good and bad points depending on the point of view [54]. However, considering the bacteria in the scalp, it may be the crucial factor that causes scalp itching.

Recently, people want chemical-free products (chemophobia). For this reason, there are people who are aiming for a no-poo, washing their heads and hair with 
water only. In contrast, as urban life becomes more common, people are concerned about the pollution and cleaning thoroughly. It is not easy to clean well when chemicals such as surfactants are not used. It is technically ironic. To solve this irony, we can suggest the following technical approach. First, only mild scalp cleaning is performed. Surfactants disturb skin barriers; hence, people with relatively weak scalp barriers are prone to irritability or itching. After that, it is appropriate to apply strengthening material to the scalp.

\section{Conclusions}

Strengthening scalp barrier function: further application of skin care ingredient:

The scalp has been of interest in areas primarily dealing with diseases such as dandruff. However, this review confirms that it is important to treat scalp barrier functions like normal facial skin. Strengthening the skin barrier improves dandruff of the scalp. Now it is necessary to expand the targeting skin barrier from face to the scalp. Effective substances or ingredients proven to strengthen the facial skin barrier may be applied to the scalp. For example, anti-aging material can be applied to alleviate scalp aging. However, it is not the answer simply to extend the substance that enhances the barrier function of skin care to the scalp. There is a huge, independent organ called hair follicle on the scalp. Hair follicles are directly linked to hair loss. Although it is a component that enhances the barrier function of the skin, there are few studies on how it affects hair follicles. Therefore, further studies on the possibility of causing hair loss should be performed before using the skin care ingredients.

For sensitive scalp care: less affective, mild formulations:

Among the external factors that cause scalp itch, the most interesting area in terms of customers and product developers will be surfactants. Damage to skin proteins and lipids by surfactants can cause skin barrier damage [55]. If the barrier is damaged or disrupted, certain stimuli are likely to penetrate the living keratinocyte of the epidermal layer. When the surfactant penetrated into the epidermal layer, keratinocytes secrete IL- $1 \alpha$ and releases various cytokines, leading to symptoms such as edema, erythema, itching, and pain [56]. In this point of view, keeping the scalp skin barrier will help resolve the customer complaint about the sensitive scalp. Because of this fact, there is a continuing need for the development of low-irritant or mild formulations. Infiltration of a single surfactant that does not form micelle can be a problem. It is necessary to use a surfactant that induces relatively less irritation, and a technical approach to induce effective micelle formation to reduce residual surfactant.

Collectively, it is important to understand the cause of grievances of customers. Knowing the factors that cause customer complaints can help researchers discover the insights of product development.

\section{Acknowledgements}

S.H. Kim, S.N. Kim, and S.H. Shin investigated and summarized the related contents. H.N. Kim gave us great inspiration to write this review. 


\section{Funding Sources}

The study was supported by grants from Amorepacific R\&D Center.

\section{Conflicts of Interest}

The authors declare no conflicts of interest regarding the publication of this paper.

\section{References}

[1] Cash, T.F. (1999) The Psychosocial Consequences of Androgenetic Alopecia: A Review of the Research Literature. British Journal of Dermatology, 141, 398-405. https://doi.org/10.1046/j.1365-2133.1999.03030.x

[2] Cash, T.F., Price, V.H. and Savin, R.C. (1993) Psychological Effects of Androgenetic Alopecia on Women: Comparisons with Balding Men and with Female Control Subjects. Journal of the American Academy of Dermatology, 29, 568-575. https://doi.org/10.1016/0190-9622(93)70223-G

[3] Schwartz, J.R., Henry, J.P., Kerr, K.M., Mizoguchi, H. and Li, L. (2015) The Role of Oxidative Damage in Poor Scalp Health: Ramifications to Causality and Associated Hair Growth. International Journal of Cosmetic Science, 37, 9-15. https://doi.org/10.1111/ics.12289

[4] Schwartz, J.R., Messenger, A.G., Tosti, A., Todd, G., Hordinsky, M., Hay, R.J., et al. (2013) A Comprehensive Pathophysiology of Dandruff and Seborrheic Dermatitis-Towards a More Precise Definition of Scalp Health. Acta Dermato-Venereologica, 93, 131-137. https://doi.org/10.2340/00015555-1382

[5] Marks, R., Pearse, A.D. and Walker, A.P. (1985) The Effects of a Shampoo Containing Zinc Pyrithione on the Control of Dandruff. British Journal of Dermatology, 112, 415-422. https://doi.org/10.1111/j.1365-2133.1985.tb02314.x

[6] Grimalt, R. (2007) A Practical Guide to Scalp Disorders. Journal of Investigative Dermatology Symposium Proceedings, 12, 10-14.

https://doi.org/10.1038/sj.jidsymp.5650048

[7] Paus, R. and Cotsarelis, G. (1999) The Biology of Hair Follicles. The New England Journal of Medicine, 341, 491-497. https://doi.org/10.1056/NEJM199908123410706

[8] Thody, A.J. and Shuster, S. (1989) Control and Function of Sebaceous Glands. Physiological Reviews, 69, 383-416. https://doi.org/10.1152/physrev.1989.69.2.383

[9] Gioti, A., Nystedt, B., Li, W., Xu, J., Andersson, A., Averette, A.F., et al. (2013) Genomic Insights into the Atopic Eczema-Associated Skin Commensal Yeast Malassezia sympodialis. MBio, 4, e00572-12. https://doi.org/10.1128/mBio.00572-12

[10] Ro, B.I. and Dawson, T.L. (2005) The Role of Sebaceous Gland Activity and Scalp Microfloral Metabolism in the Etiology of Seborrheic Dermatitis and Dandruff. Journal of Investigative Dermatology Symposium Proceedings, 10, 194-197. https://doi.org/10.1111/j.1087-0024.2005.10104.x

[11] Hoath, S.B. and Leahy, D.G. (2003) The Organization of Human Epidermis: Functional Epidermal Units and Phi Proportionality. Journal of Investigative Dermatology, 121, 1440-1446. https://doi.org/10.1046/j.1523-1747.2003.12606.x

[12] O'Goshi, K., Iguchi, M. and Tagami, H. (2000) Functional Analysis of the Stratum Corneum of Scalp Skin: Studies in Patients with Alopecia Areata and Androgenetic Alopecia. Archives of Dermatological Research, 292, 605-611. https://doi.org/10.1007/s004030000185 
[13] Pouradier, F., Liu, C., Wares, J., Yokoyama, E., Collaudin, C., Panhard, S., et al. (2017) The Worldwide Diversity of Scalp Seborrhoea, as Daily Experienced by Seven Human Ethnic Groups. International Journal of Cosmetic Science, 39, 629-636. https://doi.org/10.1111/ics.12425

[14] Elias, P.M. and Brown, B.E. (1978) The Mammalian Cutaneous Permeability Barrier: Defective Barrier Function Is Essential Fatty Acid Deficiency Correlates with Abnormal Intercellular Lipid Deposition. Laboratory Investigation, 39, 574-583.

[15] Pilgram, G.S., van der Meulen, J., Gooris, G.S., Koerten, H.K. and Bouwstra, J.A. (2001) The Influence of Two Azones and Sebaceous Lipids on the Lateral Organization of Lipids Isolated from Human Stratum Corneum. Biochimica et Biophysica Acta, 1511, 244-254. https://doi.org/10.1016/S0005-2736(01)00271-1

[16] Bacon, R.A., Mizoguchi, H. and Schwartz, J.R. (2014) Assessing Therapeutic Effectiveness of Scalp Treatments for Dandruff and Seborrheic Dermatitis, Part 1: A Reliable and Relevant Method Based on the Adherent Scalp Flaking Score (ASFS). Journal of Dermatological Treatment, 25, 232-236. https://doi.org/10.3109/09546634.2012.687089

[17] Rawlings, A.V. (2006) Ethnic Skin Types: Are There Differences in Skin Structure and Function? International Journal of Cosmetic Science, 28, 79-93. https://doi.org/10.1111/j.1467-2494.2006.00302.x

[18] Hodgins, M.B. (1998) Diseases of the Hair and Scalp. British Journal of Dermatolo$g y$, 139, 360. https://doi.org/10.1046/j.1365-2133.1998.00100.x

[19] Harding, C.R., Moore, A.E., Rogers, J.S., Meldrum, H., Scott, A.E. and McGlone, F.P. (2002) Dandruff: A Condition Characterized by Decreased Levels of Intercellular Lipids in Scalp Stratum Corneum and Impaired Barrier Function. Archives of Dermatological Research, 294, 221-230. https://doi.org/10.1007/s00403-002-0323-1

[20] Saint-Leger, D. and Leveque, J.L. (1982) A Comparative Study of Refatting Kinetics on the Scalp and Forehead. British Journal of Dermatology, 106, 669-675. https://doi.org/10.1111/j.1365-2133.1982.tb11681.x

[21] Wirth, H., Neumahr, W. and Gloor, M. (1982) On the Influence of Frequent Hair Washing on the Human Sebaceous Gland (Author's Transl.). Dermatologische Monatsschrift, 168, 75-81.

[22] Baker, H. and Kligman, A.M. (1967) Measurement of Transepidermal Water Loss by Electrical Hygrometry. Instrumentation and Responses to Physical and Chemical Insults. Archives of Dermatology, 96, 441-452.

https://doi.org/10.1001/archderm.96.4.441

[23] Ozawa, T. and Takahashi, M. (1994) Skin Hydration: Recent Advances. Acta Dermato-Venereologica. Supplementum (Stockh), 185, 26-28.

[24] Bashir, S.J., Chew, A.L., Anigbogu, A., Dreher, F. and Maibach, H.I. (2001) Physical and Physiological Effects of Stratum Corneum Tape Stripping. Skin Research and Technology, 7, 40-48. https://doi.org/10.1034/j.1600-0846.2001.007001040.x

[25] Machado, M., Salgado, T.M., Hadgraft, J. and Lane, M.E. (2010) The Relationship between Transepidermal Water Loss and Skin Permeability. International Journal of Pharmaceutics, 384, 73-77. https://doi.org/10.1016/j.ijpharm.2009.09.044

[26] Tagami, H. (2008) Location-Related Differences in Structure and Function of the Stratum Corneum with Special Emphasis on Those of the Facial Skin. International Journal of Cosmetic Science, 30, 413-434. https://doi.org/10.1111/j.1468-2494.2008.00459.x

[27] Hori, H., Moretti, G., Rebora, A. and Crovato, F. (1972) The Thickness of Human Scalp: Normal and Bald. Journal of Investigative Dermatology, 58, 396-399. 
https://doi.org/10.1111/1523-1747.ep12540633

[28] Nam, S.B., Jeon, J.Y., Hwang, S.M., Bae, Y.C. and Oh, C.G. (1999) The Comparative Histopathologic Study between the Frontal Progressive Baldness Area and the Occipital Non-Baldness Area in Korean Male Pattern Baldness. Journal of the Korean Society for Aesthetic Plastic Surgery, 5, 273-280.

[29] Liu, Y., Liu, C., Collaudin, C., Saint-Leger, D., Loussouarn, G., Kravtchenko, S., et al. (2010) Challenging the Scalp with "Dry" Wash Shampooing (DW) on Chinese Men: An in Vivo Study. International Journal of Cosmetic Science, 32, 127-133. https://doi.org/10.1111/j.1468-2494.2009.00556.x

[30] Turner, G.A., Hoptroff, M. and Harding, C.R. (2012) Stratum Corneum Dysfunction in Dandruff. International Journal of Cosmetic Science, 34, 298-306. https://doi.org/10.1111/j.1468-2494.2012.00723.x

[31] Bonnist, E.Y., Pudney, P.D., Weddell, L.A., Campbell, J., Baines, F.L., Paterson, S.E., et al. (2014) Understanding the Dandruff Scalp before and after Treatment: An in Vivo Raman Spectroscopic Study. International Journal of Cosmetic Science, 36, 347-354. https://doi.org/10.1111/ics.12132

[32] Rawlings, A.V. and Matts, P.J. (2005) Stratum Corneum Moisturization at the Molecular Level: An Update in Relation to the Dry Skin Cycle. Journal of Investigative Dermatology, 124, 1099-1110. https://doi.org/10.1111/j.1523-1747.2005.23726.x

[33] Agrawal, R. and Woodfolk, J.A. (2014) Skin Barrier Defects in Atopic Dermatitis. Current Allergy and Asthma Reports, 14, 433.

https://doi.org/10.1007/s11882-014-0433-9

[34] Mondon, P., Ringenbach, C., Doridot, E. and Genet, V. (2017) Reinforcement of Barrier Function and Scalp Homeostasis by Senkyunolide A to Fight against Dandruff. International Journal of Cosmetic Science, 39, 617-621. https://doi.org/10.1111/ics.12417

[35] Borda, L.J. and Wikramanayake, T.C. (2015) Seborrheic Dermatitis and Dandruff: A Comprehensive Review. Journal of Clinical and Investigative Dermatology, 3, 10. https://doi.org/10.13188/2373-1044.1000019

[36] Takagi, Y., Ning, X., Takahashi, A., Ishizaki, C., Nishizaka, T., Kaizu, K., et al. (2018) The Efficacy of a Pseudo-Ceramide and Eucalyptus Extract Containing Lotion on Dry Scalp Skin. Clinical, Cosmetic and Investigational Dermatology, 11, 141-148. https://doi.org/10.2147/CCID.S158428

[37] Basler, K., Bergmann, S., Heisig, M., Naegel, A., Zorn-Kruppa, M. and Brandner, J.M. (2016) The Role of Tight Junctions in Skin Barrier Function and Dermal Absorption. Journal of Controlled Release, 242, 105-118. https://doi.org/10.1016/j.jconrel.2016.08.007

[38] Furuse, M., Hata, M., Furuse, K., Yoshida, Y., Haratake, A., Sugitani, Y., et al. (2002) Claudin-Based Tight Junctions Are Crucial for the Mammalian Epidermal Barrier: A Lesson from Claudin-1-Deficient Mice. Journal of Cell Biology, 156, 1099-1111. https://doi.org/10.1083/jcb.200110122

[39] Tunggal, J.A., Helfrich, I., Schmitz, A., Schwarz, H., Gunzel, D., Fromm, M., et al. (2005) E-Cadherin Is Essential for in Vivo Epidermal Barrier Function by Regulating Tight Junctions. The EMBO Journal, 24, 1146-1156. https://doi.org/10.1038/sj.emboj.7600605

[40] Mathes, C., Brandner, J.M., Laue, M., Raesch, S.S., Hansen, S., Failla, A.V., et al. (2016) Tight Junctions Form a Barrier in Porcine Hair Follicles. European Journal of Cell Biology, 95, 89-99. https://doi.org/10.1016/j.ejcb.2015.12.001

[41] Berardesca, E., Farage, M. and Maibach, H. (2013) Sensitive Skin: An Overview. In- 
ternational Journal of Cosmetic Science, 35, 2-8.

https://doi.org/10.1111/j.1468-2494.2012.00754.x

[42] Inamadar, A.C. and Palit, A. (2013) Sensitive Skin: An Overview. The Indian Journal of Dermatology, Venereology and Leprology, 79, 9-16.

https://doi.org/10.4103/0378-6323.104664

[43] Misery, L., Sibaud, V., Ambronati, M., Macy, G., Boussetta, S. and Taieb, C. (2008) Sensitive Scalp: Does This Condition Exist? An Epidemiological Study. Contact Dermatitis, 58, 234-238. https://doi.org/10.1111/j.1600-0536.2007.01288.x

[44] Ma, L., Guichard, A., Humbert, P., Zheng, S., Tan, Y., Yu, L., et al. (2016) Evaluation of the Severity and Triggering Factors of Sensitive Scalp in Chinese Females. Journal of Cosmetic Dermatology, 15, 219-225. https://doi.org/10.1111/jocd.12203

[45] Misery, L., Rahhali, N., Ambonati, M., Black, D., Saint-Martory, C., Schmitt, A.M., et al. (2011) Evaluation of Sensitive Scalp Severity and Symptomatology by Using a New Score. The Journal of the European Academy of Dermatology and Venereology, 25, 1295-1298. https://doi.org/10.1111/j.1468-3083.2010.03968.x

[46] Bin Saif, G.A., Ericson, M.E. and Yosipovitch, G. (2011) The Itchy Scalp-Scratching for an Explanation. Experimental Dermatology, 20, 959-968.

https://doi.org/10.1111/j.1600-0625.2011.01389.x

[47] O’Neill, J.L., Chan, Y.H., Rapp, S.R. and Yosipovitch, G. (2011) Differences in Itch Characteristics between Psoriasis and Atopic Dermatitis Patients: Results of a WebBased Questionnaire. Acta Dermato-Venereologica, 91, 537-540. https://doi.org/10.2340/00015555-1126

[48] Kim, T.W., Shim, W.H., Kim, J.M., Mun, J.H., Song, M., Kim, H.S., et al. (2014) Clinical Characteristics of Pruritus in Patients with Scalp Psoriasis and Their Relation with Intraepidermal Nerve Fiber Density. Annals of Dermatology, 26, 727-732. https://doi.org/10.5021/ad.2014.26.6.727

[49] Rukwied, R., Zeck, S., Schmelz, M. and McGlone, F. (2002) Sensitivity of Human Scalp Skin to Pruritic Stimuli Investigated by Intradermal Microdialysis in Vivo. Journal of the American Academy of Dermatology, 47, 245-250. https://doi.org/10.1067/mjd.2002.120461

[50] Essick, G., Guest, S., Martinez, E., Chen, C. and McGlone, F. (2004) Site-Dependent and Subject-Related Variations in Perioral Thermal Sensitivity. Somatosensory \& Motor Research, 21, 159-175. https://doi.org/10.1080/08990220400012414

[51] Kerr, K., Schwartz, J.R., Filloon, T., Fieno, A., Wehmeyer, K., Szepietowski, J.C., et al. (2011) Scalp Stratum Corneum Histamine Levels: Novel Sampling Method Reveals Association with Itch Resolution in Dandruff/Seborrhoeic Dermatitis Treatment. Acta Dermato- Venereologica, 91, 404-408.

https://doi.org/10.2340/00015555-1073

[52] Thomas, D.S., Ingham, E., Bojar, R.A. and Holland, K.T. (2008) In Vitro Modulation of Human Keratinocyte Pro- and Anti-Inflammatory Cytokine Production by the Capsule of Malassezia Species. FEMS Immunology and Medical Microbiology, 54, 203-214. https://doi.org/10.1111/j.1574-695X.2008.00468.x

[53] Tanojo, H., Boelsma, E., Junginger, H.E., Ponec, M. and Bodde, H.E. (1998) In Vivo Human Skin Barrier Modulation by Topical Application of Fatty Acids. Skin Pharmacology and Applied Skin Physiology, 11, 87-97. https://doi.org/10.1159/000029813

[54] Smith, K.R. and Thiboutot, D.M. (2008) Thematic Review Series: Skin Lipids. Sebaceous Gland Lipids: Friend or Foe? Journal of Lipid Research, 49, 271-281. https://doi.org/10.1194/jlr.R700015-JLR200 
[55] Schepky, A.G., Holtzmann, U., Siegner, R., Zirpins, S., Schmucker, R., Wenck, H., et al. (2004) Influence of Cleansing on Stratum Corneum Tryptic Enzyme in Human Skin. International Journal of Cosmetic Science, 26, 245-253.

https://doi.org/10.1111/j.1467-2494.2004.00232.x

[56] Welss, T., Basketter, D.A. and Schroder, K.R. (2004) In Vitro Skin Irritation: Facts and Future. State of the Art Review of Mechanisms and Models. Toxicology in Vitro, 18, 231-243. https://doi.org/10.1016/j.tiv.2003.09.009 